\title{
¿Sería útil la depuración de los trastornos mentales comunes en pacientes hiperfrecuentadores de servicios de salud en cuidado primario?
}

\author{
Hernán G. Rincón-Hoyos ${ }^{1}$ \\ Mérida R. Rodríguez López ${ }^{2}$ \\ Ana Maria Villa Ruiz ${ }^{3}$ \\ Carlos Augusto Hernández ${ }^{4}$ \\ Martha Lucia Ramos ${ }^{5}$
}

\begin{abstract}
Resumen
La hiperfrecuentación de servicios de salud constituye un problema para los pacientes, la familia y las instituciones. Este trabajo busca determinar la frecuencia y las características de los trastornos mentales comunes en pacientes hiperfrecuentadores de servicios que acudieron con sintomas y signos imprecisos a una institución prestadora de servicios de cuidado primario en la ciudad de Cali (Colombia) en 2007. Método: Estudio descriptivo transversal. Mediante una encuesta telefónica, que incluyó varios módulos del instrumento PRIME MD, se detectaron los trastornos mentales más frecuentes en pacientes hiperfrecuentadores. $R e-$ sultado: Los hiperfrecuentadores de servicios son, en general, mujeres laboralmente activas, con edad promedio de 38,7 años. Acuden fundamentalmente por cefalea, pero tienen una alta prevalencia de trastornos mentales comunes (somatización, depresión y ansiedad), que no son fácilmente diagnosticados por los médicos en cuidado primario. Los valores monetarios de las actividades adicionales en salud que generan estos pacientes se atribuyen fundamentalmente a las consultas médicas y a los procedimientos que se les realizan. Conclusión: Considerar a los hiperfrecuentadores de servicios de salud como un grupo de riesgo para trastornos mentales comunes plantea su tamización como una estrategia eficiente para evitar el abuso de servicios y mejorar la satisfacción con la atención recibida.
\end{abstract}

Palabras clave: Mal uso de servicios de salud, atención primaria de salud, depresión, ansiedad, trastornos somatoformes, tamizaje.

Would the Screening of Common Mental Disorders in Primary-Care Health Services Hyper-Frequent Patients Be Useful?

1 MD, MSP. Psiquiatra de Enlace e Interconsulta, Fundación Valle de Lili. Asesor Comfandi Salud IPS. Profesor Universidades ICESI, CES y de Miami. Consultor Medicina Inteligente, Cali, Colombia.

2 MD. Medica Familiar. MSc(c) Epidemiología Comfandi Salud IPS. Profesora Pontificia Universidad Javeriana, Cali, Colombia.

3 Psicóloga, Especialista en Psicología Clínica. Psicóloga Instituto Albert Ellis de Colombia, Cali, Colombia.

4 MD, MSP., Comfandi Salud IPS, Profesor Universidad del Valle, Cali, Colombia.

5 MD. Comfandi Salud IPS, Cali, Colombia. 


\section{Abstract}

Hyper-frequentation in health services is a problem for patients, their families and the institutions. This study is aimed at determining the frequency and characteristics of common mental disorders in hyper-frequent patients showing vague symptoms and signs at a primary healthcare service during the year 2007 in the city of Cali (Colombia). Methodology: Cross sectional. The most frequent mental disorders in hyper-frequent patients were detected through a telephone interview which included several modules of the PRIME MD instrument. Results: In general, healthcare service hyper-frequenters are working women, 38,7-year old in average. Basically, the consultation is due to cephalalgia but they also exhibit a high prevalence of common mental disorders (somatization, depression and anxiety) not easily diagnosed by physicians in primary care. Expenses for additional health activities generated by these patients are attributed basically to medical consultation and required procedures. Conclusion: Considering hyper-frequenters in health care services as a risk group in terms of common mental disorders involves screening as an efficient strategy to prevent abuse in service use and to improve satisfaction with the attention received.

Key words: Health services misuse, primary health, depression, anxiety, somatoform disorders, screening.

\section{Introducción}

La hiperfrecuentación - sobreutilización o uso abusivo de servicios de salud- es una conducta en salud que se caracteriza por la demanda espontánea e innecesaria de servicios. La definición no considera las atenciones que se producen por demanda inducida
$(1,2)$ como las derivadas de procesos administrativos o por el cumplimiento de los protocolos de atención. Así mismo, se debe diferenciar el paciente hiperfrecuentador del paciente somatizador, este último sufre un trastorno mental que conlleva al uso de servicios a causa de molestias fisicas sin un motivo orgánico demostrado. No todo paciente somatizador es siempre hiperfrecuentador ni viceversa. Estos pacientes también son conocidos en Colombia como consultadores crónicos o policonsultadores.

La hiperfrecuentación de servicios genera dificultades para todos los actores participantes: el paciente y su familia, los profesionales y la institución. El problema para los pacientes y las familias incluye el gasto económico en el contexto del Sistema General de Seguridad Social en Salud (SGSSS), colombiano, debido al pago de cuotas moderadoras o copagos derivados de la atención médica. Lo anterior es acompañado por la insatisfacción del paciente con el servicio al no contar con un diagnóstico preciso con respecto a los sintomas o a la enfermedad base. De otra parte, el profesional considera por lo general, estos casos como "pacientes dificiles", quienes le generan una carga laboral y emocional, lo que puede dificultar la relación médico-paciente (3). Así mismo, es un inconveniente para las aseguradoras y para las instituciones prestadoras de salud debido al costo adicional que representan las ayudas diagnósticas, las citas médicas, 
los procedimientos y las consultas especializadas; de hecho, se ha estimado que el $10 \%$ de los gastos en salud pueden ser atribuidos a la sobreutilización de consulta (4-6).

Según el modelo teórico de uso de servicios de Aday y Andersen (1974) (7), además de las características de la prestación del servicio de salud que en algunos casos pueden generar un sobreuso de los mismos, los pacientes con trastornos mentales comunes podrían tener una percepción de necesidad de servicios superior a la de los pacientes sin estos trastornos $(1,2,8-10)$. En Colombia, en donde es muy alto el número de casos de trastornos ansiosos y depresivos, no se encontraron estudios publicados que sustenten su relación con el uso y abuso de servicios en cuidado primario ni tampoco estudios que estimen la magnitud y los costos que este problema le genera al sistema de salud.

En una institución prestadora de servicios de salud (IPS) de baja complejidad de la Ciudad de Cali, se realizó un estudio descriptivo con el objetivo de determinar la prevalencia de trastornos mentales comunes en los pacientes hiperfrecuentadores que acudieron a los servicios con sintomas y signos imprecisos. Los resultados contribuyen a posicionar el tema de la salud mental como componente estratégico para generar intervenciones enfocadas a aumentar la detección de estos trastornos e intervenir el sobreuso de servicios en instituciones de baja complejidad.

\section{Métodos}

Diseño y población de estudio: se realizó un estudio descriptivo en una población de pacientes que acudieron 7 o más veces a consulta médica general en una IPS de baja complejidad en la Ciudad de Cali durante el año 2007.

Muestra: se utilizó como fuente de información una base de datos compuesta por los registros de consultas medicas de 66 mil pacientes (23\% de la población atendida por medio de capitación) que acudieron a los servicios 7 o más veces en un año (ventana temporal: 2007). Se realizó un muestreo no probabilístico en el que se seleccionaron aquellos pacientes que acudieron a consultas y cuyas historias clinicas reportaban sintomas y signos inespecíficos sin diagnóstico confirmado de trastorno mental u orgánico de base que los explicaran. Se consideraron como causas inespecíficas aquellos diagnósticos cuya historia clínica referian: cefaleas, depresión, ansiedad, temor a estar enfermas, consulta no especificada, problemas relacionados con un horario estresante de trabajo, dificultades con la pareja o insomnio no orgánico. Se excluyeron las consultas relacionadas con la formulación de medicamentos o consultas de programa, así como los pacientes con un diagnóstico de trastorno mental severo confirmado (esquizofrenia trastorno afectivo bipolar, sindrome mental orgánico, trastorno severo 
de personalidad, retraso mental), o menores de 15 años.

Instrumentos: para la recolección de información se diseñó una encuesta que fue realizada por medio de conversaciones telefónicas e incluyó los módulos de depresión, ansiedad, somatización y salud general del cuestionario Evaluación de la atención primaria de los trastornos mentales (PRIME-MD, por sus siglas en inglés) (11). La encuesta fue realizada por dos auxiliares de enfermería entrenadas con anterioridad para la ejecución de la misma.

Procedimientos: la información fue recolectada en una base de datos. Los diagnósticos iniciales fueron agrupados de la siguiente manera: en el grupo de cefaleas se incluyeron aquellas ocasionadas por la tensión psicológica, la postraumática y las migrañas. En el de depresión se incluyeron: episodio depresivo leve, episodio depresivo moderado, episodio depresivo no especificado, otros episodios depresivos y el trastorno depresivo de la conducta. Por su parte, fueron considerados como ansiedad otros trastornos de ansiedad, trastorno de ansiedad no especificada, trastorno de ansiedad generalizada y el trastorno fóbico de ansiedad.

Debido a que el modelo estándar de oro es la evaluación clínica y a que la encuesta solo determinó la frecuencia de los sindromes mentales, se clasificaron los diagnósticos reportados en dos categorías: probables o improbables, según los criterios de la encuesta, teniendo en cuenta que la sensibilidad es menor para el módulo de somatización. A los pacientes con diagnóstico probable se les dio una capacitación sobre la demanda para el adecuado manejo clínico.

Descripción: El valor de las actividades que se facturaron a la institución fue determinado por aquellos costos que fueron atribuibles a procedimientos, consultas médicas y ayudas diganósticas, reportados en los aplicativos institucionales. En el momento de la encuesta se solicitó el consentimiento informado verbal. Para respetar la confidencialidad de los datos, los nombres de los pacientes fueron omitidos y el protocolo fue sometido y aprobado por el comité de ética institucional.

Análisis: los datos fueron procesados en el programa Epidat 3.0 para determinar las proporciones en las variables categóricas y en los promedios, acompañados de la respectiva desviación estándar (DE) para las continuas. Para las diferencias en las variables categóricas se utilizó la prueba de $\chi^{2}$ y para la comparación de promedios se usó la $t$ de Student.

\section{Resultados}

De la población total hiperfrecuentadora, el 9\% correspondió a pacientes que realizaron consultas por demanda espontánea. La muestra estuvo constituida por 1.150 pacientes de los cuales 767 fueron elegidos, lo cual corresponde al $67 \%$ 
de los seleccionados. El diagrama de flujo del estudio se muestra en la figura 1 .

Los pacientes que no fueron depurados correspondieron a aquellos que no se pudieron localizar después de varios intentos, incluso en diferentes horarios. Lo anterior se debió a la inconsistencia en los reportes telefónicos disponibles en las fuentes de información de la institución, así como a cambios de domicilio. Solamente dos pacientes se negaron a participar.
Las características sociodemográficas de la población se muestran en la tabla 1. No se observaron diferencias estadísticamente significativas en la edad de los pacientes por sexo $(p=0,0732)$, pero sí por régimen de afiliación, siendo en promedio los cotizantes de menor edad que los beneficiarios [37,5 (DE: 12,8) versus 40,9 (DE: 16,4), $p<0,001$ ]. $\mathrm{El}$ $90 \%$ de la población se encontraba entre los 15 y los 60 años.

Al analizar los diagnósticos reportados en la historia clínica

Figura 1. Diagrama de flujo del estudio.

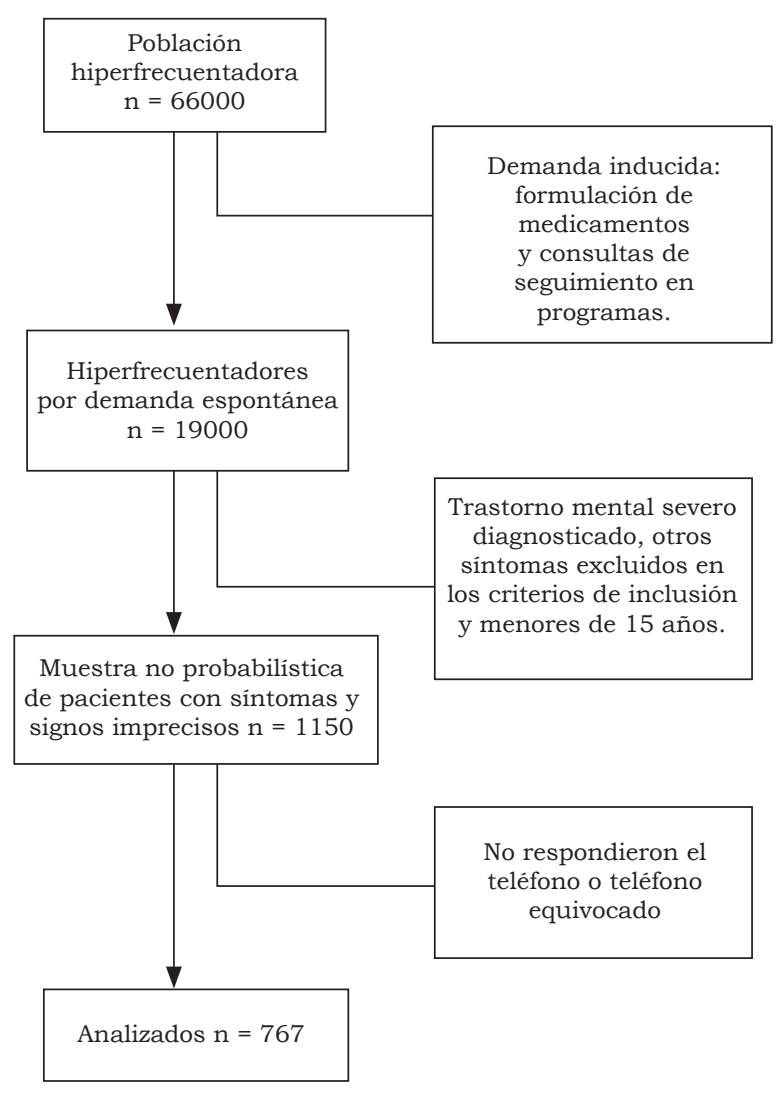


antes de realizar la encuesta telefónica, $62,8 \%$ correspondieron a sintomas de cefalea, casi $2 \%$ de los pacientes acudieron por problemas relacionados con la sexualidad y $8,4 \%$ reportaron causas no específicas.

Tabla 1. Características sociodemográficas de la población de estudio.

\begin{tabular}{|l|c|}
\hline \multicolumn{2}{|c|}{ Caracteristicas sociodemográficas } \\
$\mathbf{n}=\mathbf{7 6 7}$ \\
\hline Sexo femenino (\%) & 83,07 \\
\hline Afiliación cotizante (\%) & 64,29 \\
\hline Edad (media [DE]) & $38,67(14,29)$ \\
\hline
\end{tabular}

Al explorar la presencia de los trastornos mentales más frecuentes en el cuidado primario, se encontró que solo $5,6 \%$ había sido diagnosticado de depresión y $6,3 \%$ de ansiedad en la historia clínica; mientras que el $8,5 \%$ tenía trastornos mixtos ansioso-depresivos, solo $0,1 \%$ de los pacientes tenía registro de trastorno de somatización. Por otro lado, después de realizar la encuesta, $41,9 \%$ de los pacientes tenía síntomas de depresión o síndrome depresivo establecido. Así mismo, la ansiedad se presentó como sintoma o síndrome en $13,3 \%$ de los casos. De acuerdo con esto, $55,2 \%$ de los pacientes resultó positivo para síntomas o sindromes mentales que no habian sido reportados en la historia clínica. La presencia de estos trastornos después de realizar la encuesta se muestra en la tabla 2.

Debido a que los sintomas y signos inespecíficos podrian ser parte de los trastornos de somatización, y que este resultó ser el de mayor recurrencia después de realizada la encuesta, se evaluó la relación

Tabla 2. Distribución de los trastornos evaluados según el PRIME-MD en pacientes hiperfrecuentadores de una IPS de cuidado primario en Cali durante el año 2007 (Después de aplicar la encuesta).

\begin{tabular}{|c|l|c|}
\hline \multicolumn{2}{|c|}{$\begin{array}{c}\text { Diagnóstico después de la encuesta } \\
\text { n }=\mathbf{7 6 7}\end{array}$} & Frecuencia relativa (\%) \\
\hline \multirow{2}{*}{ Depresión } & Improbable & $79,5 \%$ \\
\cline { 2 - 3 } & Probable & $20,5 \%$ \\
\hline \multirow{2}{*}{ Ansiedad } & Improbable & $90,5 \%$ \\
\cline { 2 - 3 } & Probable & $9,52 \%$ \\
\hline \multirow{2}{*}{ Posible somatización } & Improbable & $62,3 \%$ \\
\cline { 2 - 3 } & Probable & $37,7 \%$ \\
\hline
\end{tabular}


entre este y los trastornos ansiosos depresivos (tabla 2). Los pacientes con somatización probable mostraron mayor propensión a la depresión y la ansiedad, según se observa en la figura 2 , en comparación con la prevalencia de estos trastornos en los pacientes con somatización improbable. En ambos casos las diferencias fueron estadísticamente significativas $(p<0,05)$.

En cuanto a los costos de las actividades asociados a los pacientes de la muestra, la media fue $\$ 421.200$ pesos colombianos; $25 \%$ de estos pacientes utilizaron servicios por valores superiores a $\$ 722.250$ pesos, con un caso extremo de un paciente

Figura 2. Prevalencia de trastornos de ansiedad y depresión en pacientes hiperfrecuentadores con probable diagnóstico de trastorno de somatización en una IPS de cuidado primario en Cali durante el año 2007.

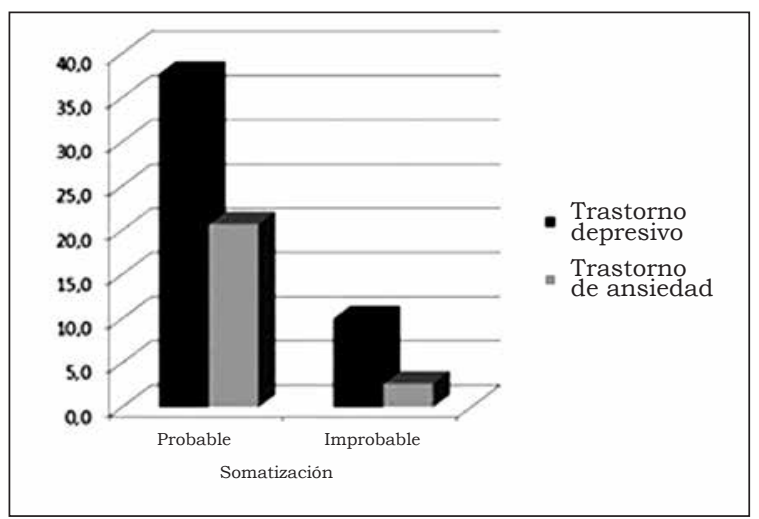

Figura 3. Distribución porcentual de los valores según los servicios utilizados por los pacientes hiperfrecuentadores en una IPS de cuidado primario en Cali durante el año 2007.

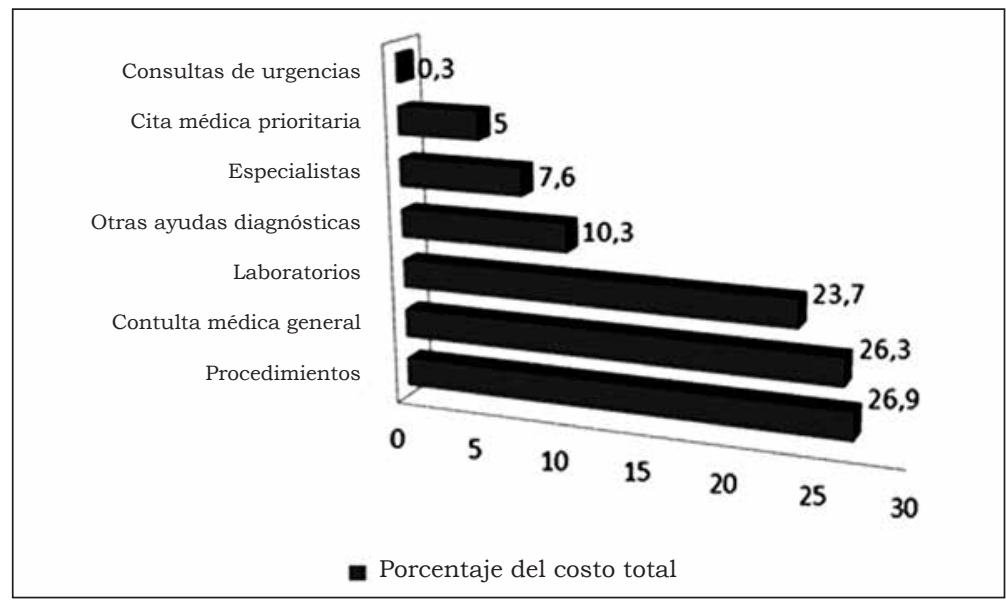


que gastó $\$ 880.580$ pesos en el año. La figura 3 expone la distribución porcentual de los valores según los servicios utilizados, la mayoría de los cuales correspondieron a las ayudas diagnósticas y a los procedimientos.

\section{Discusión}

E1 fenómeno de la hiperfrecuentación es preocupante para todo el sistema sanitario debido al impacto que causa en el paciente, las familias, las aseguradoras y las instituciones prestadoras de servicios de salud (3,4-6). Las características sociodemográficas evaluadas en este estudio mostraron que las mujeres en edad laboral que cotizan al sistema constituyen el perfil del paciente hiperfrecuentador en cuidado primario; lo cual coincide con lo descrito por algunos autores que han mostrado la relación entre la hiperfrecuentación y el sexo femenino, así como el incremento con la edad $(8,10,12,13)$. Por esta razón, algunos autores proponen que la definición del paciente hiperfrecuentador debe diferenciarse por edad y sexo, para evitar la sobredimensión del género femenino y del adulto mayor $(1,4)$.

Este hallazgo es particularmente importante porque plantea que los hiperfrecuentadores son mujeres trabajadoras, cuya productividad se ve afectada cada vez que acuden a los servicios, según sugiere la literatura; además, estas personas también tienen un mayor riesgo de pensión por discapacidad (5), aspecto que se suma a un mayor impacto económico para el SGSSS. De hecho, los hiperfrecuentadores de servicios triplican las licencias por enfermedad o pensión por invalidez en comparación con los "normofrecuentadores" y estas aumentan con cada evento vital estresante al que esté sometido el paciente (10). Así mismo, se estima que entre el 35 y el $45 \%$ del ausentismo laboral se debe a problemas de salud mental (14), lo cual hace potencialmente vulnerable a esta población según los hallazgos de este estudio.

Se he reportado que estos pacientes, quienes consumen del 10 al $16 \%$ del gasto en salud por exceso de ayudas diagnósticas, polifarmacia, citas médicas y procedimientos, demandan siete veces más consultas médicas y 2,5 veces más prescripciones (15-18). En este estudio no se realizó un análisis detallado del impacto en la unidad de pago por capitación (UPC), ni se consideraron las diferencias de los ponderadores de los grupos poblacionales. Si se asume el mayor valor de la UPC en 2007 en adultos que correspondió a las mujeres entre 15 y 44 años de edad (\$501.228) (19), y al considerar la prevalencia del $9 \%$ de hiperfrecuentadores adultos, de los cuales el $25 \%$ consume recursos por valores superiores a la UPC, se infiere que en una población hipotética de doscientos mil (200.000) afiliados que acudan a los servicios de una EPS de régimen contributivo, 4.500 
consumirán servicios por encima de este valor, lo que impacta de manera directa a las instituciones de salud. La brecha económica podría ser mayor si el crecimiento poblacional no es acompañado de intervenciones encaminadas a intervenir este comportamiento en salud.

Adicionalmente, la economía familiar se ve afectada ya que los copagos y las cuotas moderadoras, en particular de la población beneficiaria, y las incapacidades laborales de los cotizantes, se suman a otros costos indirectos como los que se atribuyen al transporte para acceder a los servicios.

Frente a lo anterior, un ensayo clínico en pacientes con síntomas imprecisos en cuidado primario demostró que una comunicación entre profesional y paciente, que incluya la discución de las preocupaciones y problemas desde la perspectiva del paciente, es clave para disminuir la ansiedad y mejorar la satisfacción con el servicio, y que estos resultados son independientes de la indicaciones de ayudas diagnósticas (20) como estrategia para calmar la ansiedad.

De otra parte, la literatura sugiere que $17 \%$ de los hiperfrecuentadores tienen al menos dos episodios de consultas con sintomas inexplicados (21); aunque no se cuantificó el uso de medicamentos en este estudio, las consultas médicas generadas y los procedimientos innecesarios podrian poner en riego la seguridad del paciente.
En este estudio se encontró que la cefalea es uno de los síntomas imprecisos más frecuentes y que además de ser parte del cortejo sintomático del trastorno de somatización, enmascara trastornos ansiosos o depresivos como ha sido demostrado en varios estudios $(22,23)$.

La prevalencia de casi el $40 \%$ de los trastornos de somatización en hiperfrecuentadores demuestra que a pesar de presentarse muchas veces de manera simultánea, no son sinónimos, lo cual destaca la distinción entre ambos términos y supone la búsqueda de otras causas más allá de este trastorno. Un estudio mostró que un $41 \%$ de los casos hiperfrecuentadores se pueden atribuir a enfermedades crónicas, un $31 \%$ a trastornos mentales y un $15 \%$ a estrés agudo y crónico. Juntos, estos tres factores explican dos tercios del fenómeno (13). Uno de los hallazgos fundamentales de este estudio fue el subregistro de los trastornos mentales, particularmente entre aquellos pacientes que acuden a consulta con síntomas imprecisos, y corrobora, junto con otros estudios (8,24-27), la conclusión de alta presencia de los mismos, en particular de depresión, entre quienes utilizan los servicios médicos (28). Estos resultados coinciden con un estudio que mostró que los médicos no diagnostican el trastorno de ansiedad y tampoco reconocen sus sintomas (29); una situación similar se reportó para el trastorno de pánico, en el cual 
también se detectó un elevado uso de servicios innecesarios $(30,31)$. Estos hallazgos refuerzan la necesidad de la detección y tratamiento de estos trastornos más allá de la atención de los eventos agudos.

En relación con la somatización, antes de hacer la encuesta se presentó solamente como diagnóstico en la historia clínica en $0,1 \%$ de los casos, mientras que posterior a la misma ascendió su probable presencia a $37,7 \%$. A su vez, la depresión pasó de una prevalencia de $5,6 \%$ a $20,5 \%$. Las diferencias para la ansiedad y los trastornos mixtos fueron menores, lo cual supone que la somatización y la depresión son los trastornos menos diagnosticados en los servicios.

El Estudio nacional de salud mental reportó prevalencias en el último año de los trastornos del estado de ánimo y de ansiedad de 6,9 y $11,7 \%$ respectivamente (32); de tal manera que la presencia de estos diagnósticos antes de realizar la encuesta fue inferior al reporte poblacional. Después de la encuesta se evidencia un comportamiento diferencial en la prevalencia de estos trastornos en los servicios en comparación con la población general, al menos en el paciente hiperfrecuentador.

La falta de integralidad del diagnostico, centrado en el síntoma físico, podría condicionar el sobreuso de servicios y el empleo indiscriminado de ayudas diagnósticas y de procedimientos innecesarios (26). Una enfermedad médica puede conducir a un trastorno mental o viceversa y con ello a la sobreutilización de servicios. Con respecto a esto, se han propuesto cinco perfiles diferentes del paciente hiperfrecuentador de servicios: (1) los pacientes con enfermedades físicas, (2) los pacientes con enfermedades psiquiátricas establecidas, (3) los pacientes en crisis, (4) los pacientes somatizadores crónicos, y (5) aquellos con múltiples problemas. La mayoría de los pacientes se encuentran en los grupos 1 y 4 (8), aunque es necesaria la evaluación de las comorbilidades que puedan influir en nuestro medio, de la cual no dio cuenta este estudio.

Un estudio reportó una asociación elevada entre los trastornos mentales y la utilización de servicios (Odds ratio (OR) ajustado por covariables de 3,1; IC95\%: 2,4-3,9) y por tanto sugirió el abordaje de los mismos mediante estrategias basadas en la familia (33). Para lograrlo, es necesario mejorar la capacidad de detección de los médicos y el trabajo en equipos transdisciplinarios que permitan no solamente intervenir el individuo, sino también su entorno.

Por otra parte, algunos estudios han mostrado que el tratamiento de la depresión en pacientes con historia de altos costos mejora los sintomas, incrementa la calidad de vida y la productividad laboral, mejora el uso de servicios y por lo tanto los costos asociados $(24,34)$; sin embargo, esto solo puede hacerse una vez aclarado el diagnóstico, frente a lo cual se ha estimado que 
los médicos prescriben solamente en el $39 \%$ de las depresiones - por mencionar uno solo de los sindromes-, este estudio plantea además que jincluso en pacientes hiperfrecuentadores el subregistro de los trastornos mentales es alto!

\section{Limitaciones}

En este estudio no fueron encuestados 383 pacientes $(13 \%$ de pérdida), sin embargo, las causas de dichas pérdidas, según lo reportado en los resultados, hacen improbable la presencia de sesgo en la selección. No se tuvieron en cuenta dentro de los criterios de inclusión a los pacientes con lumbalgia, por lo que la aplicación de estas estimaciones podría estar limitada en esta población, aunque varios reportes internacionales han dado cuenta de la asociación de los trastornos mentales con esta patología, pero no de su relación con el uso de servicios. Sin embargo, la exclusión de estos pacientes, la ausencia de datos relacionados con otros costos directos como aquellos pertenecientes a medicamentos o procedimientos y la atención de niveles superiores de complejidad incluyendo las hospitalizaciones, y el uso de recursos humanos y administrativos, así como la exclusión de los costos indirectos subestiman los valores reportados en este estudio.

La utilización del Prime MD condiciona un posible sesgo de información debido a la sensibilidad y especificidad de la prueba (11), pero al ser aplicada en una población de alta prevalencia su valor predictivo positivo aumenta. No obstante, los datos relacionados con la somatización se deben interpretar con cautela por ser el módulo con menor validez en el instrumento. Adicionalmente, puesto que la encuesta hace referencia a la presencia de los síntomas en las últimas cuatro semanas, si el periodo de tiempo transcurrido desde la última consulta a la encuesta telefónica fue superior a un mes, existe la probabilidad que algunos pacientes sin el registro del antecedente en la historia desarrollaran alguno de los trastornos de manera previa a la evaluación y no fueran consultados, por lo que es probable que se haya sobredimensionado el subregistro. Sin embargo, la historia natural de estos trastornos y la prevalencia esperada en la población que realiza una consulta de los mismos hacen que esta situación sea poco probable. Adicionalmente, se debe considerar que no siempre el subregistro es sinónimo de una falta de diagnóstico, pero que de cualquier forma es un reflejo de la escasa importancia que puede dársele a los sintomas mentales en relación con los sintomas físicos.

Por otra parte y dada la alta prevalencia de depresión y ansiedad en la población colombiana, este estudio no dio cuenta del comportamiento de estos trastornos en los grupos control que incluian pacientes con patologías crónicas o 
que no eran hiperfrecuentadores, para establecer medidas de asociación con mérito de causalidad. Otras variables como las comorbilidades físicas, el soporte social, la satisfacción del servicio, las características del profesional y la relación médicopaciente, entre otras, no fueron consideradas para este estudio.

\section{Conclusiones}

Los hiperfrecuentadores podrian constituir un grupo de alto riesgo en el que las estrategias de tamización de trastornos mentales pueden llegar a ser más eficientes que en la población general. Los resultados de este estudio establecen la relación entre los patrones de utilización y los trastornos mentales en pacientes de atención primaria, y con ello propone la necesidad de considerar en este grupo poblacional la detección y abordaje de los trastornos mentales comunes: ansiedad, depresión y somatización en las IPS de baja complejidad, como una estrategia potencialmente costo-efectiva que permita mejorar la integralidad de la atención, para lo cual es indispensable entrenar a los profesionales de estos servicios en la detección y el diagnóstico adecuado de los mismos al menos $(35,36)$. Lo anterior cobra importancia debido a los tiempos de improductividad atribuidos tanto a las consultas médicas, como a los procedimientos y ayudas diagnósticas a los que se someten, lo que condiciona además un mayor riesgo de eventos adversos.

\section{Referencias}

1. Vedsted $\mathrm{P}$, Christensen MB. Frequent attenders in general practice care: A literature review with special reference to methodological considerations Public Health. 2005;119:118-37.

2. Hughes $\mathrm{G}$. The inappropriate attender. Emerg Med J. 2008;25:468.

3. Rodríguez MR, Rincón-Hoyos HG, Velásquez JD. Evaluación psicosocial en medicina psicosomática. En: RincónHoyos HG, Velásquez JD. Medicina psicosomática y psiquiatría de enlace. Medellin (Colombia): Corporación para Investigaciones Biológicas; 2010. p. 20-29.

4. Gill D, Sharpe M. Frequent consulters in general practice: A systematic review of studies of prevalence, associations and outcome. J Psychosom Res. 1999:47:115-30.

5. Bergh $\mathrm{H}$, Baigi $\mathrm{A}$, Mansson $\mathrm{J}$, et al. Predictive factors for long-term sick leave and disability pension among frequent and normal attenders in primary health care over 5 years. Public Health. 2007; 121:25-33.

6. Smits $\mathrm{F}$, Brouwer $\mathrm{HJ}$, van Weert $\mathrm{H}$, et al. Predictability of persistent frequent attendance: a historic 3-year cohort study. Br J Gen Pract. 2009;59:114-9.

7. Aday LA, Andersen R. Framework for the study of access to medical care. Health Serv Res. 1974;9:208-20.

8. Karlsson H, Joukamaa M, Lahti I, et al. Frequent attender profiles: different clinical subgroups among frequent attender patients in primary care. $J$ Psychosom Res. 1997;42:157-66.

9. Karlsson H, Lehtinen V, Joukamaa M. Psychiatric morbidity among frequent attender patients in primary care. Gen Hosp Psychiatr. 1995;17:19-25.

10. Vedsted P, Fink $P$, Sørensen HT, et al. Physical, mental and social factors associated with frequent attendance in Danish general practice. A population- 
based cross-sectional study. Soc Sci Med. 2004;59:813-23.

11. Spitzer RL. Validation and utility of a Self-Report Version of PRIME-MD. JAMA. 1999;282:1737-44.

12. Luciano JV, Serrano A. Frequent user patients in primary care: sociodemographic profile, clinical characteristics, and a proposed new definition. Atencion Primaria. 2008;40:631-2.

13. Rodríguez C, Cebriá J, Corbella S, et al. Rasgos de personalidad y malestar psíquico asociados a los pacientes hiperfrecuentadores de consultas de atención primaria. Medifam. 2003:13:143-50.

14. Organización Mundial de la Salud (OMS). Invertir en salud mental [internet]. 2004. [citado: 15 de julio de 2012]. Disponible en: http://whqlibdoc.who. int/publications/2004/9243562576.pdf.

15. Sherbourne CD, Asch SM, Shugarman LR. Early Identification of co-occurring pain, depression and anxiety. J Gen Intern Med. 2009;24:620-5.

16. Bass CA, Bond A, Gill D, et al. Frequent attenders without organic disease in a gastroenterology clinic: Patient characteristics and health care use. Gen Hosp Psychiatr. 1999;21:30-8.

17. Robinson JO, Granfield AJ. The frequent consulter in primary medical care. J Psychosom Res. 1986;30:589600.

18. Barsky AJ, Orav EJ, Bates BW. Somatization increases medical utilization and costs independent of psychiatric and medical comorbidity. Arch Gen Psychiatry 2005;62:903-10.

19. República de Colombia, Ministerio de la Protección Social. Acuerdo 351 de 2006, Por el cual se fija el valor de la unidad de pago por capitación del plan obligatorio de salud de los regímenes contributivo y subsidiado para el año 2007 [internet]. 2007 [citado: 15 de julio de 2012]. Disponible en: http://www. minsalud.gov.co/Normatividad.

20. van Bokhoven MA, Koch $\mathrm{H}$, van der Weijden T, et al. Influence of watchful waiting on satisfaction and anxiety among patients seeking care for unexplained complaints. Ann Fam Med. 2009;7:112-20.
21. Reid S, Wessely S, Crayford TIM, et al. Frequent attenders with medically unexplained symptoms: service use and costs in secondary care. Br J Psychiatr. 2002;180:248-53.

22. Jyväsjärvi S, Joukamaa M, Väisänen $E$, et al. Somatizing frequent attenders in primary health care. J Psychosom Res. 2001;50:185-92.

23. Williams ERL, Guthrie E, MackwayJones K, et al. Psychiatric status, somatisation, and health care utilization of frequent attenders at the emergency department: A comparison with routine attenders. J Psychosom Res. 2001;50:161-7.

24. Katzelnick DJ, Kobak KA, Greist JH, et al. Effect of primary care treatment of depression on service use by patients with high medical expenditures. Psychiatr Serv. 1997;48:59-64.

25. Muñoz RA, McBride ME, Brnabic AJ, et al. Major depressive disorder in Latin America: The relationship between depression severity, painful somatic symptoms, and quality of life. J Affect Dis. 2005;86:93-8.

26. Menchetti M, Cevenini N, De Ronchi D, et al. Depression and frequent attendance in elderly primary care patients. Gen Hosp Psychiatr. 2006;28:119-24.

27. Dueñas HJ, Dwight T, McBride M, et al. Effectiveness of antidepressants in the treatment of major depressive disorder in Latin America. Int J Psychiatr Clin Pract. 2007;11:129-39.

28. Lloyd K, Jenkins R. The economics of depression in primary care. Department of Health initiatives. $\mathrm{Br} \mathrm{J}$ Psychiatry Suppl. 1995;27:60-2.

29. Calleo J, Stanley MA, Greisinger A, et al. Generalized anxiety disorder in older medical patients: diagnostic recognition, mental health management and service utilization. J Clin Psychol Med Settings. 2009;16:178-85.

30. Roy-Byrne PP, Russo J, Dugdale DC, et al. Undertreatment of panic disorder in primary care: role of patient and physician characteristics. J Am Board Fam Pract. 2002;15:443-50.

31. Roy-Byrne, PP, Stein MB, Russo J, et al. Panic disorder in the primary care setting: comorbidity, disability, service 
utilization, and treatment. J Clin Psychiatry. 1999;60:492-9.

32. República de Colombia, Ministerio de la Protección Social (Minprotección). Tercer estudio nacional de salud mental y consumo de sustancias psicoactivas Colombia 2003. Cali: Minprotección, FES; 2005.

33. Bellón JA, Delgado A, Luna JD, et al. Psychosocial and health belief variables associated with frequent attendance in primary care. Psychol Med. 1999;29:1347-57.
34. Bellon Saameno JA, Rodríguez Bayon A. What can really be done to reduce the number of clinic visits by the frequent-user patient? Atencion Primaria. 2008;40:591-3.

35. Lefevre F, Reifler D, Lee P, et al. Screening for undetected mental disorders in high utilizers of primary care services.J Gen Intern Med. 1999;14:425-31.

36. Halverson J, Chan C. Screening for psychiatric disorders in primary care. WMJ. 2004;103:46-51.

Conflictos de interés: Los autores manifiestan que no tienen conflictos de interés en este artículo.

Recibido para evaluación: 16 de agosto de 2012 Aceptado para publicación: 17 de septiembre de 2012

Correspondencia

Hernán G. Rincón-Hoyos

Psiquiatria de Enlace

Fundación Valle del Lili

Calle 50 N. ${ }^{\circ} 118 A-68$

hernangrincon@gmail.com 